\title{
The Impact of Sound Exposure on Heart Rate Variability in Adolescent Students
}

\section{VETERNIK ${ }^{1}$, I. TONHAJZEROVA ${ }^{2,3}$, J. MISEK $^{1}$, V. JAKUSOVA ${ }^{4}$, H. HUDECKOVA ${ }^{4}$, J. JAKUS ${ }^{1}$}

${ }^{1}$ Department of Medical Biophysics, Jessenius Faculty of Medicine in Martin, Comenius University in Bratislava, Martin, Slovakia, ${ }^{2}$ Department of Physiology, Jessenius Faculty of Medicine in Martin, Comenius University in Bratislava, Martin, Slovakia, ${ }^{3}$ Biomedical Center Martin, Jessenius Faculty of Medicine in Martin, Comenius University in Bratislava, Martin, Slovakia, ${ }^{4}$ Department of Public Health, Jessenius Faculty of Medicine in Martin, Comenius University in Bratislava, Martin, Slovakia

Received February 5, 2018

Accepted March 29, 2018

On-line July 25, 2018

\section{Summary}

Previous studies of physiological responses to music and noise showed the effect on the autonomic nervous system. The heart rate variability (HRV) has been used to assess the activation of the sympathetic and the parasympathetic nervous systems. The present study was aimed to examine HRV with exposure to four sine-wave pure tones $(20 \mathrm{~Hz}, 50 \mathrm{~Hz}, 2 \mathrm{kHz}$ and $15 \mathrm{kHz})$ in an environment where the sound intensity exceeded level $65 \mathrm{~dB}$ (A-weighted). The participants (20 adolescent girls) were lying in supine position during exposure protocol divided into 6 periods, the first time with generated sounds and the second time without sounds. In the protocol without sound exposure, the low frequency band of the HRV spectrum was increased compared to the basal state before examination (period_1: $6.05 \pm 0.29 \mathrm{~ms}^{2}$ compared to period_5: $\left.6.56 \pm 0.20 \mathrm{~ms}^{2}, \mathrm{p}<0.05\right)$. The significant increase of root Mean Square of the Successive Differences (rMSSD, period_1: $4.09 \pm 0.16 \mathrm{~s}$ compared to period_6: $4.33 \pm 0.12 \mathrm{~s}, \mathrm{p}<0.05)$ and prolongation of $\mathrm{R}$ to $\mathrm{R}$ peak (RR) interval (period_1: $889 \pm 30 \mathrm{~ms}$ compared to period_5: $973 \pm 30 \mathrm{~ms}, \mathrm{p}<0.001$ ) were observed in the protocol without sound exposure comparing to the protocol with sound exposure where only bradycardia was observed. Contrary to rather polemical data in literature our pilot study suggests that sounds (under given frequencies) have no impact on the heart rate variability and cardiac autonomic regulation.

\author{
Key words \\ Autonomic nervous system • Heart rate variability • Cardiac \\ regulation • Sound • Noise
}

\section{Corresponding author}

J. Jakus, Department of Medical Biophysics, Jessenius Faculty of Medicine in Martin, Comenius University in Bratislava, Mala Hora 4, 03601 Martin, Slovakia. E-mail: jakus@jfmed.uniba.sk

\section{Introduction}

Listening to music is usually a very pleasurable experience for the humans (Dube and Le Bel 2003). Music can be defined as an organization of the tones over the time with a strong impact on the allostatic regulatory mechanisms including autonomic nervous system (ANS) (Riganello et al. 2015). Several studies showed a correlation between listening to music, noise or pure sounds and activity of cardiac autonomic regulation (Perez-Lloret et al. 2014, Orini et al. 2010, Iwanaga et al. 2005).

The cardiac function is extremely sensitive to autonomic regulatory inputs. The heart rate variability (HRV) - perpetual heart rate oscillations around its mean value - reflects the activities of sympathetic and vagal components of the ANS on the cardiac sinus node (Sztajzel 2004). Notably, the heart rate is controlled by a complex regulatory network including a highly 
integrated neural system known as the central autonomic network (CAN) through which the brain controls visceromotor, neuroendocrine, and behavioral responses (Lane et al. 2009, Benarroch 1993). Furthermore, the Polyvagal Theory (Porges 2007, Porges 1995) describes the integration of neurocardiac regulation through nervus vagus with the neural regulation of the middle ear muscles mediated by V and VII cranial nerves to extract human voice from background sounds as an important component of Social Engagement System that is crucial for physiological flexibility and adaptability (Porges 2009, Porges 2007, Porges 2003). Several studies reported cardiac-linked vagal dysregulation in clinical disorders characterized by problems in affective and social behavior such as depression or ADHD (Tonhajzerova et al. 2016, Tonhajzerova et al. 2010, Tonhajzerova et al. 2009). Importantly, the deficits in auditory processing can be manifested in expressive and receptive language skills leading to down-regulated Social Engagement System, such as in autism (Porges 2013a, Porges et al. 2013b). In this aspect, the effect of sounds with various frequencies on HRV was not sufficiently clarified in healthy humans. Sim et al. (2015) studied the impact of noise on HRV in men, with a focus on the noise type and noise intensity. They proved impact of noise on ANS concluding that the effect on the HRV activity might differ according to the noise type. For example, Iwanaga et al. (2005) examined the effect of repetitive exposure to sedative and excitative music on HRV. This study revealed a decrease of cardiac vagal regulation during excitative music; in contrast, another study (Da Silva et al. 2014) concluded that music with different tempos (no music, classical baroque and heavy metal music) did not influence the cardiac autonomic regulation indexed by HRV.

The question regarding the impact of the distinct tone frequency on HRV is still unresolved. The $20 \mathrm{~Hz}$ tone frequency is usually considered to be a threshold of humans hearing (Møller and Pedersen 2004) and also lower limit of spectrum analyzing or sound recording devices. The sound range of $2-5 \mathrm{kHz}$ is the most sensitive frequency band for a human ear (Kanders et al. 2017, Javorka 2001) while the sounds with frequencies around $15 \mathrm{kHz}$ are more difficult to hear, however, can still cause auditory perception (Bak and Rosniak-Bak 2013). Based on these studies, we hypothesized that exposure to low frequency sounds $(20 \mathrm{~Hz}$ and $50 \mathrm{~Hz})$ could increase the parasympathetic nerve activity and decrease the sympathetic nerve activity and exposure to mid and high frequency sounds $(2 \mathrm{kHz}$ and $15 \mathrm{kHz})$ could result in increase of the sympathetic nerve activity and decreased the parasympathetic nerve activity. We aimed to identify the impact of four sine-wave pure tones on ANS by recording of HRV in the environment where the sound intensity exceeded level $65 \mathrm{~dB}$ (A-weighted), which is known to have a physiological impact on the human body (Žiaran 2008). To the best of our knowledge, this is the first study to examine the impact of repetitive pure tones on the cardiac autonomic regulation quantified by a shortterm HRV analysis.

\section{Methods}

\section{Subjects}

Twenty adolescent girls in good health condition studying at Viliam Pauliny-Tóth grammar school (average age: $17.4 \pm 0.2$ years, body mass index (BMI): $20.9 \pm 0.3 \mathrm{~kg} / \mathrm{m}^{2}$ ) were enrolled in this study. Exclusion criteria were following: acute or chronic diseases potentially influencing HRV (flu, endocrinological or cardiovascular diseases), medications taking, obesity and underweight. Limits for BMI (weight $(\mathrm{kg}) /$ height $^{2}\left(\mathrm{~m}^{2}\right)$ ) were determined according to the international standards (centile range within $10^{\text {th }}$ and $90^{\text {th }}$ centile curves valid for girls at the age of 3-18 years) (Cole et al. 2007) and by BMI reference values adjusted for the Slovak population (Ševčíková et al. 2004). The same participants were also used for control measurements without sound exposure. The participants were asked to avoid smoking and drinking alcoholic and caffeinated beverages at least $24 \mathrm{~h}$ before the trial.

\section{Ethics Statement}

The study was approved by the Ethics Committee of Jessenius Faculty of Medicine in Martin, Comenius University in Bratislava in accordance with the 1964 Helsinki declaration and its later amendments. All the participants and their parents were carefully instructed about the study protocol. The written informed consent was admitted prior to the examination.

\section{Sound exposure protocol}

All 20 participants were exposed to four different pure tones generated by functional signal generator Agilent N9310A (Agilent Technologies, Santa Clara, CA). Subjects were examined in a quiet room with the same light intensity under standard conditions (temperature: $22-23{ }^{\circ} \mathrm{C}$ ). Before examination, the body 
temperature, height, weight and blood pressure were measured (Misek et al. 2018). After $10 \mathrm{~min}$ adaptation to the conditions in the examination room, the participants were instructed to lie down comfortably on a special bed and rest in the supine position. To avoid visual stimuli eye mask was fit on participant's head. The exposure protocol was performed in 6 successive periods (Period 1S, Period 2S, Period 3S, Period 4S, Period 5S, Period $6 \mathrm{~S}$ ), where each was at least $5 \mathrm{~min}$ long (required for the HRV analysis in time domain). 300 RR long interval was taken for short-term HRV analysis in the frequency domain (Camm et al. 1996).

During the first adaptation period Period_1S the participants were exposed only to room noise without any generated sounds $\left(\mathrm{L}_{\mathrm{Aeq}}=32.7 \pm 0.7 \mathrm{~dB} ; \mathrm{L}_{\text {Aeq }}\right.$, equivalent continuous sound level with A-frequency weighting), during Period_2S, Period_3S, Period_4S and Period_5S the participants were exposed to continuous sine wave tones with frequencies $20 \mathrm{~Hz}$ (Period_2S, $\mathrm{L}_{\text {Aeq }}=65.5 \pm$ $0.1 \mathrm{~dB}$ as averaged through all participants), $50 \mathrm{~Hz}$ (Period_3S, $\left.\quad \mathrm{L}_{\text {Aeq }}=65.7 \pm 0.1 \mathrm{~dB}\right), \quad 2 \mathrm{kHz} \quad$ (Period_4S, $\mathrm{L}_{\mathrm{Aeq}}=65.7 \pm 0.2 \mathrm{~dB}$ ) and $15 \mathrm{kHz}$ (Period_5S, $\mathrm{L}_{\text {Aeq }}=64.9 \pm 0.5 \mathrm{~dB}$ ) during $5 \mathrm{~min}$. During the last Period_6S the participants were exposed only to room noise again without generated sounds $\left(\mathrm{L}_{\mathrm{Aeq}}=32.3 \pm 0.9 \mathrm{~dB}\right)$ (Fig. 1). The room noise (in our circumstances) represented sounds with $\mathrm{L}_{\text {Aeq }} \leq 35 \pm 0.7 \mathrm{~dB}$ which could be generated by any activities or objects happening or placed outside the examining room but the sounds could be transferred through the walls or closed windows to our examining room.

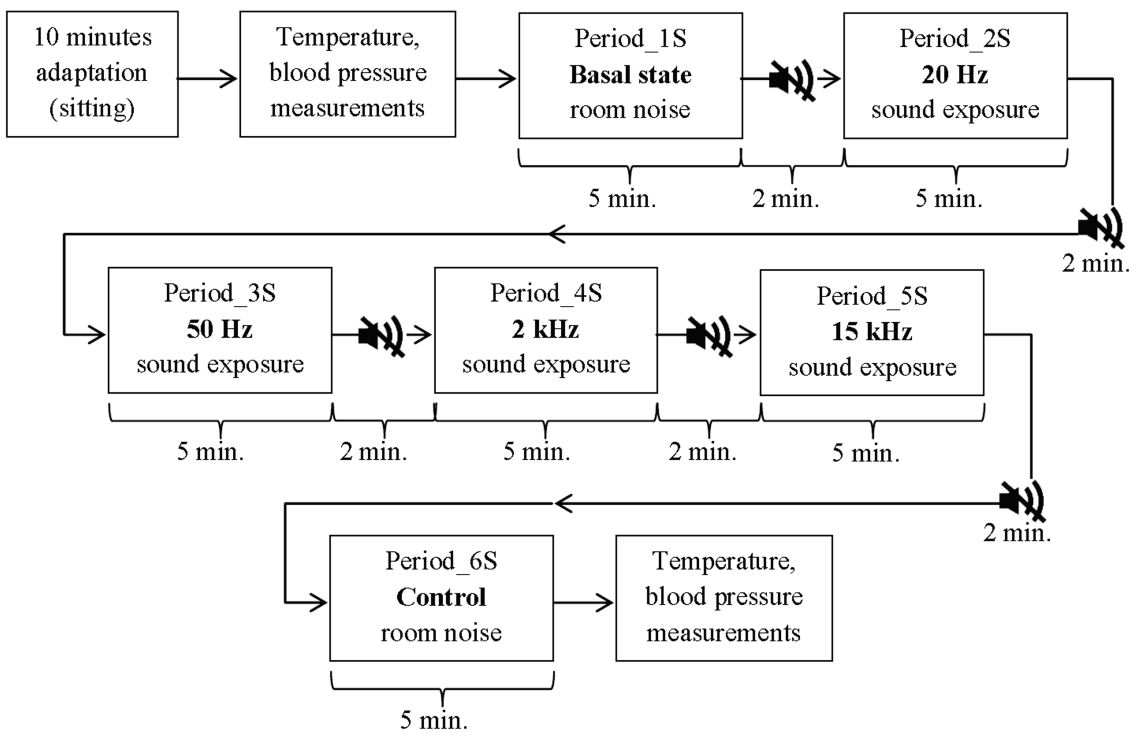

Fig. 1. Diagram of protocol with sound exposure.

All the $\mathrm{L}_{\text {Aeq }}$ values were being measured for 5 min by integrating-averaging sound level meter Brüel \& Kjær Type 2240 (Class 1 precision, Brüel \& Kjær, Nærum, Denmark). Soundmeter was mounted on a tripod and located at the distance of $10 \mathrm{~cm}$ from the right ear at the level of ears of participants. The minimal distance from the walls and large reflexive surfaces was $3 \mathrm{~m}$ according to the recommendations for free field sound measurement and STN EN ISO 9612 standard. Sounds with the frequencies $20 \mathrm{~Hz}$ and $50 \mathrm{~Hz}$ were reproduced by 15" active subwoofer Ibiza Sound SUB15A (Lotronic s.a., Saintes, Belgium) and sounds with frequencies $2 \mathrm{kHz}$ and $15 \mathrm{kHz}$ were reproduced by loudspeakers Creative SBS A320 (Creative Technology Ltd, Singapore, Malaysia). For the verification of these reproduced sounds, they were recorded retrospectively by a capacitor microphone BLUE Yeti
Blackout (Blue Microphones, Westlake Village, CA) connected to a soundcard in the personal computer. Subsequently, the recorded sounds were processed by fast Fourier transformation and the spectrum was checked for requested frequencies. Subwoofer and loudspeakers were placed $1 \mathrm{~m}$ from the proband's head. After each measurement period the sound exposure was interrupted for $2 \mathrm{~min}$. The body temperature and blood pressure were measured again after sixth period. Six-period measurement protocol was repeated (after 1 week) in all 20 participants during the second examination. However, the sound exposure $(20 \mathrm{~Hz}, 50 \mathrm{~Hz}, 2 \mathrm{kHz}, 15 \mathrm{kHz})$ was substituted by room noise without generated sounds marked as Period_1N, Period_2N, Period_3N, Period_4N, Period_5N and Period_6N $\left(\mathrm{L}_{\mathrm{Aeq}}=35 \pm 0.7 \mathrm{~dB}\right)$ that served as control measurements (Fig. 2). 


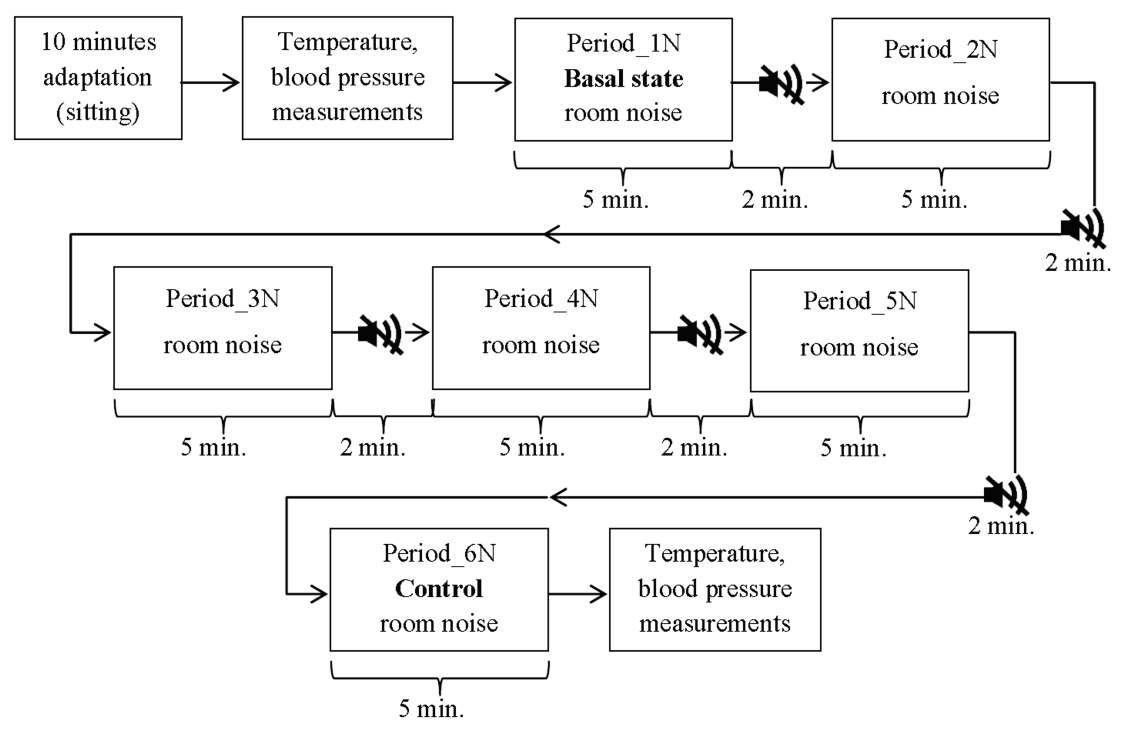

Fig. 2. Diagram of protocol without sound exposure.

\section{HRV measurement}

HRV is very sensitive to any excitement which can modify HRV parameters, heart rate and respiration rate. Therefore, no speaking was permitted during measurement periods (Bernardi et al. 2000). All students underwent one-lead electrocardiograph (ECG) recording during the sound exposure as well as without sound exposure periods. A chest belt with two electrodes was situated on the thoracic segment of midclavicular lines. ECG signal was recorded telemetrically using HRV system DiANS PF8 (Dimea Group, Olomouc, the Czech Republic). All recordings were subjected to HRV manual filtration to identify and eliminate artifacts and extrasystoles of the heart electric activity according to the generally accepted recommendations (Camm et al. 1996). In ECG records lasting $300 \mathrm{RR}$ intervals, a $256 \mathrm{RR}$ intervals window was used for fast Fourier transformation spectral analysis to determine two distinct regions: the low frequency band (LF: 0.04-0.15 Hz), mainly reflecting the baroreflex activity mediated by both the sympathetic and parasympathetic regulation, and the high frequency band (HF: $0.15-0.5 \mathrm{~Hz}$ ), reflecting predominantly the activity of the parasympathetic nervous system (Sim et al. 2015). The following HRV parameters were evaluated: RR intervals [ms], root Mean Square of the Successive Differences (rMSSD, [s]) indicating parasympathetic nerve activity, spectral power in low frequency (LF-HRV $\left[\mathrm{ms}^{2}\right]$ ) and high-frequency bands (HF-HRV $\left[\mathrm{ms}^{2}\right]$ ) reflecting ANS activity and respiratory rate (brpm, breaths per minute).

\section{Statistical analysis}

Data processing was carried out using MS Office Excel and GraphPad InStat (La Jolla, CA, USA) software. All data were analyzed for the Gaussian/nonGaussian distribution with the Kolmogorov-Smirnov test.
The parameters with the non-Gaussian distribution (rMSSD, LF, HF) were log-transformed to obtain the parameters with Gaussian distribution. Consecutively, Student's paired test was used for comparison of the side differences. The probabilities $\mathrm{p}<0.05$ were considered significant. The data were expressed as a mean \pm SEM.

\section{Results}

\section{Physiological parameters}

The significant decrease of the heart rate was observed in the protocol with sound exposure (before Period_1S: $74 \pm 3 \mathrm{bpm}$ vs. after Period_6S: $65 \pm 2 \mathrm{bpm}$, $\mathrm{p}<0.001$, bpm, beats per minute) and also during the protocol without sound exposure (before Period_1N: $75 \pm 3$ bpm vs. after Period_6N: 67 \pm 2 bpm, p $<0.01)$. Systolic blood pressure significantly decreased during the protocol without sound exposure (before Period_1N: $121 \pm 2 \mathrm{~mm} \mathrm{Hg}$ vs. after Period_6N: $116 \pm 2 \mathrm{~mm} \mathrm{Hg}$, $\mathrm{p}=0.05)$. The respiratory rate was not changed during all periods of protocol with sound exposure as well as during protocol without sound exposure.

\section{HRV parameters}

The duration of RR intervals was significantly prolonged in Period_2S, Period_3S, Period_4S, Period_5S and Period_6S during sound exposure protocol (compared to Period_1S, $\mathrm{p}<0.05$ ), as well as in Period_3N, Period_4N, Period_5N and Period_6N during protocol without sound exposure (compared to Period_1N, $\mathrm{p}<0.05$ ) (Table 1 and Table 2). rMSSD gradually increased from baseline in Period_1N to Period_5N towards the signify-cant difference in Period_6N (Period_1N vs. Period_6N, p<0.05). 
Table 1. Dynamics of HRV parameters in protocol with sound exposure.

\begin{tabular}{|c|c|c|c|c|c|c|}
\hline \multirow{2}{*}{$\begin{array}{l}\text { Physiological } \\
\text { parameter }\end{array}$} & \multicolumn{6}{|c|}{ Sound exposure } \\
\hline & Period_1S & $\begin{array}{c}\text { Period_2S } \\
(20 \mathrm{~Hz})\end{array}$ & $\begin{array}{c}\text { Period_3S } \\
(50 \mathrm{~Hz})\end{array}$ & $\begin{array}{c}\text { Period_4S } \\
(2 \mathrm{kHz})\end{array}$ & $\begin{array}{c}\text { Period_5S } \\
(15 \text { kHz) }\end{array}$ & Period_6S \\
\hline$R R[\mathrm{~ms}]$ & $888 \pm 30$ & $909 \pm 32 *$ & $937 \pm 35^{* *}$ & $944 \pm 30 * * *$ & $952 \pm 32 * * *$ & $938 \pm 24 * * *$ \\
\hline$r M S S D[\ln (s)]$ & $4.20 \pm 0.11$ & $4.18 \pm 0.11$ & $4.22 \pm 0.12$ & $4.17 \pm 0.13$ & $4.21 \pm 0.12$ & $4.23 \pm 0.12$ \\
\hline$L F-H R V\left[\ln \left(m s^{2}\right)\right]$ & $5.91 \pm 0.17$ & $5.82 \pm 0.16$ & $6.20 \pm 0.16$ & $6.26 \pm 0.11$ & $6.14 \pm 0.20$ & $6.29 \pm 0.17^{*}$ \\
\hline$H F-H R V\left[\ln \left(m s^{2}\right)\right]$ & $7.42 \pm 0.2$ & $7.30 \pm 0.22$ & $7.37 \pm 0.23$ & $7.26 \pm 0.24$ & $7.36 \pm 0.22$ & $7.40 \pm 0.22$ \\
\hline
\end{tabular}

Data are presented as mean $\pm \mathrm{SEM}$; RR, $\mathrm{R}$ to $\mathrm{R}$ peak interval [ms]; rMSSD, root Mean Square of the Successive Differences [s]; LF-HRV, low frequency band in spectral analysis $\left[\mathrm{ms}^{2}\right]$; HF-HRV, high frequency band in spectral analysis $\left[\mathrm{ms}^{2}\right] ; \mathrm{p}$ values are compared to Period_1S values; $* p<0.05, * * p<0.01, * * * p<0.001$.

Table 2. Dynamics of HRV parameters in protocol without sound exposure.

\begin{tabular}{lcccccc}
\hline \multirow{2}{*}{$\begin{array}{l}\text { Physiological } \\
\text { parameter }\end{array}$} & Period_1N & Period_2N & Period_3N & Period_4N & Period_5N & Period_6N \\
\cline { 2 - 7 } & $889 \pm 30$ & $919 \pm 34$ & $947 \pm 32^{*}$ & $951 \pm 31^{* *}$ & $973 \pm 30^{* * *}$ & $979 \pm 26^{* * *}$ \\
$R R[m s]$ & $4.09 \pm 0.16$ & $4.13 \pm 0.16$ & $4.23 \pm 0.14$ & $4.27 \pm 0.13$ & $4.29 \pm 0.14$ & $4.33 \pm 0.12^{*}$ \\
$r M S S D[\ln (s)]$ & $6.05 \pm 0.29$ & $6.16 \pm 0.19$ & $6.20 \pm 0.18$ & $6.49 \pm 0.16$ & $6.56 \pm 0.20^{*}$ & $6.47 \pm 0.18$ \\
$L F-H R V\left[\ln \left(m s^{2}\right)\right]$ & $7.20 \pm 0.30$ & $7.25 \pm 0.30$ & $7.36 \pm 0.25$ & $7.42 \pm 0.22$ & $7.46 \pm 0.25$ & $7.56 \pm 0.23$ \\
$H F-H R V\left[\ln \left(\mathrm{ms}^{2}\right)\right]$ & & & & & & \\
\hline
\end{tabular}

Data are presented as mean \pm SEM; RR, R to R peak interval [ms]; rMSSD, root Mean Square of the Successive Differences [s]; LF-HRV, low frequency band in spectral analysis $\left[\mathrm{ms}^{2}\right] ;$ HF-HRV, high frequency band in spectral analysis $\left[\mathrm{ms}^{2}\right] ; \mathrm{p}$ values are compared to Period_1N values; $* p<0.05, * * p<0.01, * * * p<0.001$.

\section{Discussion}

Contrary to the former hypothesis, these findings revealed no significant effect of sounds under different given frequencies on the cardiac autonomic regulation indexed by the HRV parameters. In the protocol without sound exposure, the LF-HRV was increased in the Period_5N compared to basal state in Period_1N. The interpretation of the LF component is controversial and it is considered as a marker of baroreflex activity determined by both sympathetic and parasympathetic regulation (Sim et al. 2015). Thus, we suggest dominant parasympathetic regulatory inputs on the heart rate control as physiological adaptive response to the supine position. Moreover, our results demonstrated the gradual RR intervals prolongation indicating a bradycardic reaction in both protocols - with sound and without sound exposure. In accordance to previous suggestion, it could reflect a shift of resting cardiac autonomic dynamic balance towards parasympathetic augmentation indexed also by the significant increase of the time domain HRV parameter rMSSD in the protocol without sound exposure. Several explanations are assumed.

The supine position represents physiological mechanism where the cardiac-linked vagal efferent inputs are maximal. In this aspect, the short-term HRV is mediated predominantly by the parasympathetic modulation, the sympathetic nervous system is slow to follow beat-to-beat heart rate oscillations (Javorka 2008). Thus, it could explain the increase of the cardiac parasympathetically-linked regulation represented by rMSSD observed in our study during the protocol without sound exposure.

On the contrary, the protocol with sound exposure did not show significant changes in HRV parasympathetic parameters, so we assume a modification of the cardiac autonomic regulation in the supine position as an effect of the sound exposure. Furthermore, the interaction between the cardiac autonomic regulation and auditory stimulation is based on the acoustic startle reflex evoked by loud sounds (around $110 \mathrm{~dB}$ ) influencing the arterial blood pressure and heart rate as a response to abrupt loud auditory stimulus (Chikahisa et al. 2007). These stimuli are transferred through outer and middle 
ear on the hair cells in the inner ear where they create a receptor potential transferred on an afferent nerve fiber. The receptor potential causes synapse potentials in receptive terminals of neurons in Corti ganglion and they are transferred to the brain through auditory nerve in a form of the neural excitation (Javorka 2001).

Unlike the previous findings, this study was designed to assess the impact of distinct sound frequencies, rather than the intensity of generated sounds. The major studies investigated the impact of either various types of music or noise. Music is the organization of various pure tones over the time with the evidenced impact on autonomic nervous system (Riganello et al. 2015). The term noise refers to sound with multiple frequencies that is loud or unpleasant or that causes disturbance. The novelty of this study is the examination of pure tones with low and high frequencies and their impact on cardiac autonomic regulation indexed by the HRV parameters. Unlike our former expectations, the exposure to mid and high sound frequencies $(2 \mathrm{kHz}$ and $15 \mathrm{kHz}$ ) did not result in the increased sympathetic activity and decreased parasympathetic activity. However, significant increase of the rMSSD parameter and prolongation of $R R$ intervals were observed as a physiological adaptive response to the supine position (lasting almost $1 \mathrm{~h}$ ) in the protocol without sound exposure contrary to the protocol with sound exposure where only bradycardia was observed. Furthermore, RR intervals parameter can be an indicator for both parasympathetic and sympathetic activity. To the best of our knowledge, this is the first study that examined the effect of sine wave pure tones on the autonomic nerve system in humans.

However, this study has several limitations. The first one is relatively small number of participants, therefore, these results should be validated in a large group. Furthermore, this study included only women, therefore, the gender differences should be investigated to elucidate this question. Regarding HRV parameters, we did not include the LF/HF ratio that is extensively discussed. Despite the fact that the previous studies indicated that the LF-HRV could be used as a possible marker of cardiac sympathetic regulation (Malliani et al. 1991, Pagani et al. 1986), recent evidence showed different results: 1) LF-HRV is substantially blocked by vagal, but not sympathetic blockade, 2) physiological and psychological manipulations increasing sympathetic outflow often do not raise the LF-HRV, but reduce it, 3) pharmacological interventions, e.g. isoprenaline, inducing sympathetic activation do not enhance LF-HRV, 4) there is a lack of associations between LF-HRV and valid indicators of sympathetic cardiac regulation, such as pre-ejection period (Reyes Del Paso 2013). Moreover, the mathematical expression of sympathovagal balance by LF/HF ratio includes the possibility that change of either the numerator or the denominator could result in interpretation that reduction of HF with no change in LF indicates a shift toward sympathetic predominance and reduction of LF with no change in HF express a shift toward vagal predominance (Billman 2013, Eckberg 1997). For these reasons, we did to not include the LF/HF in this study. Nevertheless, our study suggests that sounds with frequencies $20 \mathrm{~Hz}, 50 \mathrm{~Hz}, 2 \mathrm{kHz}$ and $15 \mathrm{kHz}$ have no impact on the cardiac autonomic regulation analyzed by the heart rate variability. We suggest that this finding could contribute to better understanding of the "soundautonomic regulation" in healthy subjects providing thus important information for clinical practice (e.g. musicotherapy). Further studies will be required to ascertain the role of various sound frequencies on ANS.

\section{Conflict of Interest}

There is no conflict of interest.

\section{Acknowledgements}

This study was supported by the Slovak Research and Development Agency under the contract No. APVV0189-11" (prof. Jakus), by the project VEGA 1/0166/17, VEGA 1/0072/16, VEGA $1 / 0253 / 15$ and VEGA 1/0044/18. The authors appreciate Mgr. H. Habinakova, $\mathrm{PhD}$. for recruitment of the students.

\section{References}

BAK M, ROSNIAK-BAK K: Upper frequency limit of hearing - a new screening method or an illusion? J Biomed Eng Technol 1: 31-35, 2013.

BENARROCH EE: The central autonomic network: functional organization, dysfunction, and perspective. Mayo Clin Proc 68: 988-1001, 1993. 
BERNARDI L, WDOWCZYK-SZULC J, VALENTI C, CASTOLDI S, PASSINO C, SPADACINI G, SLEIGHT P: Effects of controlled breathing, mental activity and mental stress with or without verbalization on heart rate variability. J Am Coll Cardiol 35: 1462-1469, 2000.

BILLMAN GE: The LF/HF ratio does not accurately measure cardiac sympatho-vagal balance. Front Physiol 4: 26, 2013.

CAMM A, MALIK M, BIGGER J, GÜNTER B, CERUTTI S, CHOEN R: 1996. Heart rate variability: standards of measurement, physiological interpretation and clinical use. Task force of the European Society of Cardiology and the North American Society of Pacing and Electrophysiology. Circulation 93: 1043-1065, 1996.

CHIKAHISA S, SANO A, KITAOKA K: Anxiolytic effect of music depends on ovarian steroid in female mice. Behav Brain Res 179: 50-59, 2007.

COLE TJ, FLEGAL KM, NICHOLLS D, JACKSON AA: Body mass index cut offs to define thinness in children and adolescents: international survey. Br Med J 335: 194-201, 2007.

DA SILVA AG, GUIDA HL, ANTÔNIO AM, MARCOMINI RS, FONTES AM, CARLOS DE ABREU L, ROQUE AL, SILVA SB, RAIMUNDO RD, FERREIRA C, VALENTI VE: An exploration of heart rate response to differing music rhythm and tempos. Complement Ther Clin Pract 20: 130-134, 2014.

DUBE L, LE BEL J: The content and structure of laypeople's concept of pleasure. Cogn Emot 17: 263-295, 2003.

ECKBERG DL: Sympathovagal balance: A critical appraisal. Circulation 96: 3224-3232, 1997.

IWANAGA M, KOBAYASHI A, KAWASAKI C: Heart rate variability with repetitive exposure to music. Biol Psychol 70: 61-66, 2005.

JAVORKA K: Heart Rate Variability: Mechanisms, Evaluation, Clinical Use. (In Slovak). Osveta, Martin, 2008, 204 p.

JAVORKA K: Medical Physiology: Textbook for Medical Faculties. (In Slovak). Osveta, Martin, 2001, 679 p.

KANDERS K, LORIMER T, GOMEZ F, STOOP R: Frequency sensitivity in mammalian hearing from a fundamental nonlinear physics model of the inner ear. Sci Rep 7: 9931, 2017.

LANE RD, MCRAE K, REIMAN EM, CHEN K, AHERN GL, THAYER JF: Neural correlates of heart rate variability during emotion. Neuroimage 44: 213-222, 2009.

MALLIANI A, PAGANI M, LOMBARDI F, CERUTTI S: Cardiovascular neural regulation explored in the frequency domain. Circulation 84: 482-492, 1991.

MISEK J, BELYAEV I, JAKUSOVA V, TONHAJZEROVA I, BARABAS J, JAKUS J: Heart rate variability affected by radiofrequency electromagnetic field in adolescent students. Bioelectromagnetics 39: 277-288, 2018.

MØLLER H, PEDERSEN CS: Hearing at low and infrasonic frequencies. Noise Health 6: 37-57, 2004.

ORINI M, BAILÓN R, ENK R, KOELSCH S, MAINARDI L, LAGUNA P: A method for continuously assessing the autonomic response to music-induced emotions through HRV analysis. Med Biol Eng Comput 48: 423-433, 2010.

PAGANI M, LOMBARDI F, GUZZETTI S, RIMOLDI O, FURLAN R, PIZZINELLI P, SANDRONE G, PEREZLLORET S, DIEZ J, DOMÉ MN, DELVENNE AA, BRAIDOT N, CARDINALI DP, VIGO DE: Effects of different "relaxing" music styles on the autonomic nervous system. Noise Health 16: 279-284, 2014.

PORGES SW: A psychophysiology of developmental disabilities: A personal and historical perspective. Am J Intellect Dev Disabil 118: 416-418, 2013a.

PORGES SW, MACELLAIO M, STANFILL SD, MCCUE K, LEWIS GF, HARDEN ER, HANDELMAN M, DENVER J, BAZHENOVA OV, HEILMAN KJ: Respiratory sinus arrhythmia and auditory processing in autism: Modifiable deficits of an integrated social engagement system? Int $J$ Psychophysiol 88: 261-270, $2013 b$.

PORGES SW: Orienting in a defensive world: Mammalian modifications of our evolutionary heritage: A polyvagal theory. Psychophysiology 32: 301-318, 1995.

PORGES SW: Social engagement and attachment. Ann N Y Acad Sci 1008: 31-47, 2003.

PORGES SW: The polyvagal perspective. Biol Psychol 74: 116-143, 2007.

PORGES SW: The polyvagal theory: New insights into adaptive reactions of the autonomic nervous system. Cleve Clin $J$ Med 76: 86-90, 2009. 
REYES DEL PASO GA, LANGEWITZ W, MULDER LJ, VAN ROON A, DUSCHEK S: The utility of low frequency heart rate variability as an index of sympathetic cardiac tone: a review with emphasis on a reanalysis of previous studies. Psychophysiology 50: 477-487, 2013.

RIGANELLO F, CORTESE MD, ARCURI F, QUINTIERI M, DOLCE G: How can music influence the autonomic nervous system response in patients with severe disorder of consciousness? Front Neurosci 9: 461, 2015.

ŠEVČÍKOVÁ L, NOVÁKOVÁ J, HAMADE J: Centile graphs and antropometric indicators. (In Slovak) Telesný vývoj detí a mládeže v SR: výsledky VI. celoštátneho prieskumu v roku 2001: 6-13, 2004.

SIM CS, SUNG JH, CHEON SH, LEE JM, LEE JW, LEE J: The effects of different noise types on heart rate variability in men. Yonsei Med J 56: 235-243, 2015.

SZTAJZEL J: Heart rate variability: a noninvasive electrocardiographic method to measure the autonomic nervous system. Swiss Med Wkly 134: 514-522, 2004.

TONHAJZEROVA I, FARSKY I, MESTANIK M, VISNOVCOVA Z, MESTANIKOVA A, HRTANEK I, ONDREJKA I: Symbolic dynamics of heart rate variability - a promising tool to investigate cardiac sympathovagal control in attention deficit/hyperactivity disorder (ADHD)? Can J Physiol Pharmacol 94: 579-587, 2016.

TONHAJZEROVA I, ONDREJKA I, ADAMIK P, HRUBY R, JAVORKA M, TRUNKVALTEROVA Z, MOKRA D, JAVORKA K: Changes in the cardiac autonomic regulation in children with attention deficit hyperactivity disorder (ADHD). Indian J Med 130: 44-50, 2009.

TONHAJZEROVA I, ONDREJKA I, JAVORKA K, TURIANIKOVA Z, FARSKY I, JAVORKA M: Cardiac autonomic regulation is impaired in girls with major depression. Prog Neuropsychopharmacol Biol Psychiatry 34: 613-618, 2010.

ŽIARAN S: Protection of Human Beings Against Vibration and Noise. (In Slovak) Slovak University of Technology, Bratislava, 2008, 264 p. 